\title{
Variational approach to second-order impulsive dynamic equations on time scales
}

\author{
Victoria Otero-Espinar ${ }^{1 *}$ and Tania Pernas-Castaño ${ }^{1,2}$
}

\section{"Correspondence:}

mvictoria.otero@usc.es

'Departamento de Análise

Matemática, Universidade de

Santiago de Compostela, Santiago

de Compostela, Galicia 15782, Spain

Full list of author information is

available at the end of the article

\begin{abstract}
The aim of this paper is to employ variational techniques and critical point theory to prove some conditions for the existence of solutions to a nonlinear impulsive dynamic equation with homogeneous Dirichlet boundary conditions. Also, we are interested in the solutions of the impulsive nonlinear problem with linear derivative dependence satisfying an impulsive condition.
\end{abstract}

MSC: 34B37; 34N05

Keywords: impulsive dynamic equations; second-order boundary value problem; variational techniques; critical point theory; time scales

\section{Introduction}

This paper is concerned with the existence of solutions of second-order impulsive dynamic equations on time scales. More precisely, we consider the following boundary value problem:

$$
(P) \quad\left\{\begin{array}{l}
-u^{\Delta \Delta}(t)+\lambda u^{\sigma}(t)=f\left(t, u^{\sigma}(t)\right) ; \quad \Delta \text {-a.e. } t \in[0, T]_{\mathbb{T}}^{\kappa^{2}}, \\
u(0)=0=u(T), \\
u^{\Delta}\left(t_{j}^{+}\right)-u^{\Delta}\left(t_{j}^{-}\right)=I_{j}\left(u\left(t_{j}^{-}\right)\right), \quad j=1,2, \ldots, p,
\end{array}\right.
$$

where the impulsive points $t_{j} \in J$ are right-dense points in an arbitrary time scale $\mathbb{T}$, with $t_{0}=0<t_{1}<t_{2}<\cdots<t_{p}<t_{p+1}=T$. Here $f:[0, T]_{\mathbb{T}} \times \mathbb{R} \rightarrow \mathbb{R}$ and $I_{j}: \mathbb{R} \rightarrow \mathbb{R}, j=1, \ldots, p$, are continuous functions.

It is well known that the theory of impulsive dynamic equations provides a natural framework for mathematical modeling of many real world phenomena. The impulsive effects exist widely in many evolution processes in which their states are changed abruptly at certain moments of time.

Applications of impulsive dynamic equations arise in biology (biological phenomena involving thresholds), medicine (bursting rhythm models), pharmacokinetics, mechanics, engineering, chaos theory, etc. As a consequence, there has been a significant development in impulse theory in recent years. We can see some general and recent works on the theory of impulsive differential equations; see [1-9] and the references therein.

For a second-order dynamic equation, we usually consider impulses in the position and velocity. However, in the motion of spacecraft, one has to consider instantaneous impulses depending on the position that result in jump discontinuities in velocity, but with no 
change in position. The impulses only on the velocity occur also in impulsive mechanics. An impulsive problem with impulses in the derivative is considered in [10].

Moreover, we are interested in the solutions of the impulsive nonlinear problem in time scale with derivative dependence satisfying an impulsive condition. We can see, for example, recent works on the theory of impulsive differential equations in $[1,3,6,8,11]$.

There have been several approaches to studying the solutions of impulsive dynamic equations on time scales, such as the method of lower and upper solutions, fixed-point theory [12-14]. Sobolev spaces of functions on time scales, which were first introduced in [15], opened a very fruitful new approach in the study of dynamic equations on time scales: the use of variational methods in the context of boundary value problems on time scales (see $[16,17])$ or in second-order Hamiltonian systems [18]. Moreover, the study of the existence and multiplicity of solutions for impulsive dynamic equations on time scales has also been done by means of the variational method (see, for example, $[19,20]$ ).

The aim of this paper is to use variational techniques and critical point theory to derive the existence of multiple solutions to $(P)$; we refer the reader to [21-24] for a broad introduction to dynamic equations on time scales and to $[25,26]$ for variational methods and critical point theory.

The paper is organized as follows. In Section 2 we gather together essential properties about Sobolev spaces on time scales proved in $[15,27,28]$ which one needs to read this paper.

The goal of Section 3 is to exhibit the variational formulation for the impulsive Dirichlet problem. As we will see, all these problems can be understood and solved in terms of the minimization of a functional, usually related to the energy, in an appropriate space of functions. The results presented in the part where we address the linear problem are basic but crucial to revealing that a problem can be solved by finding the critical points of a functional. Moreover, we prove some sufficient conditions for the existence of at least one positive solution to $(P)$.

To finish, in Section 4, we present an impulsive nonlinear problem with linear derivative dependence. We transform the problem into an equivalent one that has no dependence on the derivative, and then we prove that the problem has at least one solution. Also, with additional conditions in nonlinearities and impulse functions, we can show the existence of at least two solutions by using the mountain pass theorem.

\section{Preliminaries}

Let $\mathbb{T}$ be an arbitrary time scale. We assume that $\mathbb{T}$ has the topology that it inherits from the standard topology on $\mathbb{R}$. Assume that $a<b$ are points in $\mathbb{T}$ and define the time scale interval $[a, b]_{\mathbb{T}}=\{t \in \mathbb{T}: a \leq t \leq b\}$. We denote $J^{0}=[a, b)_{\mathbb{T}}$.

Below we set out some results proved in $[15,27]$ about Sobolev spaces on time scales.

Definition 2.1 Let $p \in \overline{\mathbb{R}}$ be such that $p \geq 1$ and $u: J \rightarrow \overline{\mathbb{R}}$. We say that $u$ belongs to $W_{\Delta}^{1, p}(J)$ if and only if $u \in L_{\Delta}^{p}\left(J^{0}\right)$, and there exists $g: J^{\kappa} \rightarrow \overline{\mathbb{R}}$ such that $g \in L_{\Delta}^{p}\left(J^{0}\right)$ and

$$
\int_{J^{0}}\left(u \cdot \varphi^{\Delta}\right)(s) \Delta s=-\int_{J^{0}}\left(g \cdot \varphi^{\sigma}\right)(s) \Delta s \quad \forall \varphi \in \mathcal{C}_{0, \mathrm{rd}}^{1}\left(J^{k}\right)
$$

with

$$
\mathcal{C}_{0, \mathrm{rd}}^{1}\left(J^{k}\right)=\left\{u: J \rightarrow \mathbb{R}: u \in \mathcal{C}_{\mathrm{rd}}^{1}\left(J^{k}\right), u(a)=u(b)=0\right\},
$$


and $\mathcal{C}_{\mathrm{rd}}^{1}\left(J^{\kappa}\right)$ is the set of all continuous functions on $J$ such that they are $\Delta$-differentiable on $J^{\kappa}$ and their $\Delta$-derivatives are rd-continuous on $J^{\kappa}$.

Theorem 2.1 Assume that $p \in \overline{\mathbb{R}}$ and $p \geq 1$. The set $W_{\Delta}^{1, p}(J)$ is a Banach space with the norm defined for every $x \in W_{\Delta}^{1, p}(J)$ as

$$
\|x\|_{W_{\Delta}^{1, p}}:=\|x\|_{L_{\Delta}^{p}}+\left\|x^{\Delta}\right\|_{L_{\Delta}^{p}}
$$

Moreover, the set $H_{\Delta}^{1}(J):=W_{\Delta}^{1,2}(J)$ is a Hilbert space with the inner product given for every $(x, y) \in H_{\Delta}^{1}(J) \times H_{\Delta}^{1}(J)$ by

$$
(x, y)_{H_{\Delta}^{1}}:=(x, y)_{L_{\Delta}^{2}}+\left(x^{\Delta}, y^{\Delta}\right)_{L_{\Delta}^{2}} .
$$

Proposition 2.1 Assume that $p \in \overline{\mathbb{R}}$ with $p \geq 1$, then there exists a constant $K>0$, only dependent on $b-a$, such that the inequality

$$
\|x\|_{\mathcal{C}())} \leq K \cdot\|x\|_{W_{\Delta}^{1, p}}
$$

holds for all $x \in W_{\Delta}^{1, p}(J)$, and hence the immersion $W_{\Delta}^{1, p}(J) \hookrightarrow \mathcal{C}(J)$ is continuous.

Definition 2.2 Let $p \in \mathbb{R}$ be such that $p \geq 1$, define the set $W_{0, \Delta}^{1, p}(J)$ as the closure of the $\operatorname{set} \mathcal{C}_{0, \mathrm{rd}}^{1}\left(J^{\kappa}\right)$ in $W_{\Delta}^{1, p}(J)$. We define $H_{0, \Delta}^{1}(J):=W_{0, \Delta}^{1,2}(J)$.

The spaces $W_{0, \Delta}^{1, p}(J)$ and $H_{0, \Delta}^{1}(J)$ are endowed with the norm induced by $\|\cdot\|_{W_{\Delta}^{1, p}}$, defined in (2.1), and the inner product induced by $(\cdot, \cdot)_{H_{\Delta}^{1}}$, defined in (2.2). These spaces satisfy the following properties.

Proposition 2.2 (Poincare's inequality) Let $p \in \mathbb{R}$ be such that $p \geq 1$. Then there exists $a$ constant $L>0$, only dependent on $b-a$, such that

$$
\|u\|_{W_{\Delta}^{1, p}} \leq L \cdot\left\|u^{\Delta}\right\|_{L_{\Delta}^{p}} \quad \forall u \in W_{0, \Delta}^{1, p}(J) .
$$

Proposition 2.3 (Corollary 3.3 in [27]) If $u \in H_{0, \Delta}^{1}(J)$, then

$$
\int_{a}^{b}\left(u^{\sigma}\right)^{2}(t) \Delta t \leq \frac{1}{\lambda_{1}} \int_{a}^{b}\left(u^{\Delta}\right)^{2}(t) \Delta t
$$

holds, where $\lambda_{1}$ is the smallest positive eigenvalue of problem $-u^{\Delta \Delta}(t)=\lambda u^{\sigma}(t) ; t \in J^{\kappa^{2}}$ and $u(a)=0=u(b)$.

In the Sobolev space $H_{0, \Delta}^{1}(J)$ with $a=0$ and $b=T$, consider the inner product

$$
(u, v)=\int_{0}^{T} u^{\Delta}(t) v^{\Delta}(t) \Delta t
$$

inducing the norm $\|\cdot\|$. 
It is the consequence of Poincare's inequality that

$$
\|u\|_{H_{0, \Delta}^{1}(J)} \leq\|u\|_{W_{\Delta}^{1,2}(J)} \leq c\left\|u^{\Delta}\right\|_{L_{\Delta}^{2}\left(J^{0}\right)} \equiv c\|u\|
$$

and

$$
\|u\| \leq\|u\|_{W_{\Delta}^{1,2}(J)} \leq 2\|u\|_{H_{0, \Delta}^{1}(J)}
$$

\section{Variational formulation of $(P)$ and existence results}

Firstly, to show the variational structure underlying an impulsive dynamic equation, we consider the lineal problem

$$
(L P) \quad\left\{\begin{array}{l}
-u^{\Delta \Delta}(t)+\lambda u^{\sigma}(t)=h(t), \quad \Delta \text {-a.e. } t \in J^{\kappa^{2}}, \\
u^{\Delta}\left(t_{j}^{+}\right)-u^{\Delta}\left(t_{j}^{-}\right)=d_{j}, \quad j=1,2, \ldots, p, \\
u(0)=u(T)=0,
\end{array}\right.
$$

where we consider $J$ with $a=0$ and $b=T$ and $d_{j}, j=1, \ldots, p$, are fixed constants.

Suppose that $u \in \mathcal{C}_{\mathrm{rd}}(J)$ is such that $u(0)=0=u(T)$. Moreover, assume that for every $j=0,1, \ldots, p, u_{j}:=\left.u\right|_{\left(t_{j}, t_{j+1}\right)}$ is such that $u_{j} \in H_{\Delta}^{2}\left(t_{j}, t_{j+1}\right)$.

Definition 3.1 We say that $u$ is a classical solution of $(L P)$ if the limits $u^{\Delta}\left(t_{j}^{+}\right)$and $u^{\Delta}\left(t_{j}^{-}\right)$ exist for every $j=1,2, \ldots, p$ and it satisfies the equation on $(L P)$ for $\Delta$-almost everywhere $\left(\Delta\right.$-a.e.) $t \in J^{\kappa^{2}}$.

Take $v \in H_{0, \Delta}^{1}(J)$, multiply the equation by $v^{\sigma}$ and integrate between 0 and $T$ :

$$
-\int_{0}^{T} u^{\Delta \Delta} v^{\sigma}+\lambda \int_{0}^{T} u^{\sigma} v^{\sigma}=\int_{0}^{T} h v^{\sigma}
$$

Taking into account that $v(0)=0=v(T)$ and integrating by parts, we get

$$
\begin{aligned}
-\int_{0}^{T} u^{\Delta \Delta} v^{\sigma} & =-\sum_{j=0}^{p} \int_{t_{j}}^{t_{j+1}} u^{\Delta \Delta} v^{\sigma}=\sum_{j=0}^{p}\left[-\left.\left(u^{\Delta} v\right)\right|_{t_{j}^{+}} ^{t_{j+1}^{-}}+\int_{t_{j}}^{t_{j+1}} u^{\Delta} v^{\Delta}\right] \\
& =\sum_{j=1}^{p} d_{j} v\left(t_{j}\right)+\int_{0}^{T} u^{\Delta} v^{\Delta} .
\end{aligned}
$$

Hence,

$$
\int_{0}^{T} u^{\Delta} v^{\Delta}+\lambda \int_{0}^{T} u^{\sigma} v^{\sigma}=\int_{0}^{T} h v^{\sigma}-\sum_{j=1}^{p} d_{j} v\left(t_{j}\right) .
$$

We define the bilinear form $a: H_{0, \Delta}^{1}(J) \times H_{0, \Delta}^{1}(J) \rightarrow \mathbb{R}$ by

$$
a(u, v)=\int_{0}^{T} u^{\Delta} v^{\Delta}+\lambda \int_{0}^{T} u^{\sigma} v^{\sigma},
$$


and the linear operator $l: H_{0, \Delta}^{1}(J) \rightarrow \mathbb{R}$ by

$$
l(v)=\int_{0}^{T} h v^{\sigma}-\sum_{j=1}^{p} d_{j} v\left(t_{j}\right) .
$$

Thus, the concept of weak solution for the impulsive problem $(L P)$ is a function $u \in$ $H_{0, \Delta}^{1}(J)$ such that $a(u, v)=l(v)$ is valid for any $v \in H_{0, \Delta}^{1}(J)$.

We can prove that $a$ defined by (3.1) and $l$ defined by (3.2) are continuous, and, from Proposition 2.3, that $a$ is coercive if $\lambda>-\lambda_{1}$.

Consider $\varphi: H_{0, \Delta}^{1}(J) \rightarrow \mathbb{R}$ defined by

$$
\varphi(v)=\frac{1}{2} a(v, v)-l(v)=\frac{1}{2} \int_{0}^{T}\left(v^{\Delta}\right)^{2}+\frac{\lambda}{2} \int_{0}^{T}\left(v^{\sigma}\right)^{2}-\int_{0}^{T} h v^{\sigma}+\sum_{j=1}^{p} d_{j} v\left(t_{j}\right) .
$$

We can deduce the following regularity properties which allow us to assert that the solutions to $(L P)$ are precisely the critical points of $\varphi$.

Lemma 3.1 The following statements are valid.

1. $\varphi$ is differentiable at any $u \in H_{0, \Delta}^{1}(J)$ and

$$
\left(\varphi^{\prime}(u), v\right)=\int_{0}^{T} u^{\Delta} v^{\Delta}+\lambda \int_{0}^{T} u^{\sigma} v^{\sigma}-\int_{0}^{T} h v^{\sigma}+\sum_{j=1}^{p} d_{j} v\left(t_{j}\right)=a(u, v)-l(v) .
$$

2. If $u \in H_{0, \Delta}^{1}(J)$ is a critical point of $\varphi$ defined by (3.3), then $u$ is a weak solution of the impulsive problem $(L P)$.

We will use the following result in linear functional analysis, which ensures the existence of a critical point of $\varphi$.

Theorem 3.1 (Lax-Milgram theorem) Let $H$ be a Hilbert space and let $a: H \times H \rightarrow \mathbb{R}$ be a bounded bilinear form. If $a$ is coercive, i.e., there exists $\alpha>0$ such that $a(u, u) \geq \alpha\|u\|^{2}$ for every $u \in H$, then for any $\sigma \in H^{\prime}$ (the conjugate space of $H$ ) there exists a unique $u \in H$ such that

$$
a(u, v)=\langle\varphi, v\rangle, \quad v \in H .
$$

Moreover, if a is also symmetric, then the functional $J: H \rightarrow \mathbb{R}$ defined by

$$
J(v)=\frac{1}{2} a(u, v)-\langle\varphi, v\rangle,
$$

attains its minimum at $u$.

By the Lax-Milgram theorem, we obtain the following result.

Theorem 3.2 If $\lambda>-\lambda_{1}$ then the problem $(L P)$ has a weak solution $u \in H_{0, \Delta}^{1}(J)$ for any $h \in L_{\Delta}^{2}\left(J^{0}\right)$. Moreover, $u \in H_{\Delta}^{2}(J)$ and $u$ is a classical solution and $u$ minimizes the functional (3.3), and hence it is a critical point of $\varphi$. 


\subsection{Impulsive nonlinear problem}

We consider the nonlinear Dirichlet problem

$$
(P) \quad\left\{\begin{array}{l}
-u^{\Delta \Delta}(t)+\lambda u^{\sigma}(t)=f\left(t, u^{\sigma}(t)\right) ; \quad \Delta \text {-a.e. } t \in J^{\kappa^{2}}, \\
u^{\Delta}\left(t_{j}^{+}\right)-u^{\Delta}\left(t_{j}^{-}\right)=I_{j}\left(u\left(t_{j}^{-}\right)\right), \quad j=1,2, \ldots, p, \\
u(0)=0=u(T) .
\end{array}\right.
$$

We assume that $\lambda>-\lambda_{1}$.

A weak solution of $(P)$ is a function $u \in H_{0, \Delta}^{1}(J)$ such that

$$
\int_{0}^{T} u^{\Delta} v^{\Delta}+\lambda \int_{0}^{T} u^{\sigma} v^{\sigma}=-\sum_{j=1}^{p} I_{j}\left(u\left(t_{j}^{-}\right)\right) v\left(t_{j}\right)+\int_{0}^{T} f\left(t, u^{\sigma}(t)\right) v^{\sigma}(t) \Delta t
$$

for every $v \in H_{0, \Delta}^{1}(J)$.

We now consider the functional

$$
\begin{aligned}
\varphi(u) & =\frac{1}{2} a(u, u)-l(u) \\
& =\frac{1}{2} \int_{0}^{T}\left(u^{\Delta}\right)^{2}+\frac{\lambda}{2} \int_{0}^{T}\left(u^{\sigma}\right)^{2}+\sum_{j=1}^{p} \int_{0}^{u\left(t_{j}\right)} I_{j}(t) d t-\int_{0}^{T} F\left(t, u^{\sigma}(t)\right) \Delta t,
\end{aligned}
$$

where $F(t, u):=\int_{0}^{u} f(t, x) d x$.

One can deduce, from the properties of $H, f$ and $I_{j}$, the following regularity properties of $\varphi$.

Proposition 3.1 The functional $\varphi$ defined by (3.4) is continuous, differentiable, and weakly lower semi-continuous. Moreover, the critical points of $\varphi$ are weak solutions of $(P)$.

Theorem 3.3 Suppose that $f$ is bounded and that the impulsive functions $I_{j}$ are bounded. Then there is a critical point of $\varphi$, and $(P)$ has at least one solution.

Proof Take $M>0$ and $M_{j}>0, j=1,2, \ldots, p$, such that

$$
|f(t, u)| \leq M \quad \forall(t, u) \in[0, T]_{\mathbb{T}} \times \mathbb{R}
$$

and

$$
\left|I_{j}(u)\right| \leq M_{j} \quad \forall u \in \mathbb{R}, j=1,2, \ldots, p .
$$

Using that $\lambda>-\lambda_{1}$, there exists $\alpha>0$ such that for any $u \in H_{0, \Delta}^{1}(J)$

$$
\begin{aligned}
\varphi(u) & \geq \frac{\alpha}{2}\|u\|^{2}+\sum_{j=1}^{p} \int_{0}^{u\left(t_{j}\right)} I_{j}(t) d t-\int_{0}^{T} F\left(t, u^{\sigma}(t)\right) \Delta t \\
& \geq \frac{\alpha}{2}\|u\|^{2}-\sum_{j=1}^{p} M_{j}\left|u\left(t_{j}\right)\right|-M \int_{0}^{T}\left|u^{\sigma}(t)\right| \Delta t .
\end{aligned}
$$


Thus, using Proposition 2.1, (2.3) and $m=\max _{j=1, \ldots, p}\left\{M, M_{j}\right\}$, we have

$$
\begin{aligned}
\varphi(u) & \geq \frac{\alpha}{2}\|u\|^{2}-m\left(\sum_{j=1}^{p}\left|u\left(t_{j}\right)\right|-\int_{0}^{T}\left|u^{\sigma}(t)\right| \Delta t\right) \\
& \geq \frac{\alpha}{2}\|u\|^{2}-m\left(p\|u\|_{\mathcal{C}(J)}+T\|u\|_{\mathcal{C}(J)}\right) \equiv \frac{\alpha}{2}\|u\|^{2}-m \rho\|u\|_{\mathcal{C}(J)} \\
& \geq \frac{\alpha}{2}\|u\|^{2}-m \rho K\|u\|_{H_{0, \Delta}^{1}} \geq \frac{\alpha}{2}\|u\|^{2}-m \rho K c\|u\|,
\end{aligned}
$$

where $\rho=p+T$.

This implies that $\lim _{\|u\| \rightarrow \infty} \varphi(u)=+\infty$, and $\varphi$ is coercive. Hence (Th. 1.1 of [26]), $\varphi$ has a minimum, which is a critical point of $\varphi$.

Theorem 3.4 Suppose that $f$ is sublinear and the impulsive functions $I_{j}$ have sublinear growth. Then there is a critical point of $\varphi$ and $(P)$ has at least one solution.

Proof Let $a, b, a_{j}, b_{j}>0$, and $\gamma, \gamma_{j} \in[0,1), j=1,2, \ldots, p$, such that

$$
|f(t, u)| \leq a+b|u|^{\gamma} \quad \text { and } \quad\left|I_{j}(u)\right| \leq a_{j}+b_{j}|u|^{\gamma_{j}} \quad \forall t \in[0, T]_{\mathbb{T}}, u \in \mathbb{R} .
$$

Again, using that $\lambda>-\lambda_{1}$, Proposition 2.1, (2.3) and $m=\max _{j=1, \ldots, p}\left\{a, a_{j}\right\}, \widetilde{m}=\max _{j=1, \ldots, p}\{b$, $b_{j}$ \}, we have

$$
\varphi(u) \geq \frac{\alpha}{2}\|u\|^{2}-\beta\|u\|-\delta\|u\|^{\gamma+1}
$$

where $\beta=m \rho K c$ and $\delta=\tilde{m} \rho K^{\gamma+1} c^{\gamma+1}$.

Since $\gamma+1<2$, then $\lim _{\|u\| \rightarrow \infty} \varphi(u)=+\infty$ for every $u \in H_{0, \Delta}^{1}(J)$.

\section{Impulsive nonlinear problem with linear derivative dependence}

Consider the following problem:

$$
(N P) \quad\left\{\begin{array}{l}
-u^{\Delta \Delta}(t)+g(t) u^{\Delta}(\sigma(t))+\lambda u^{\sigma}(t)=f\left(t, u^{\sigma}(t)\right) ; \quad \Delta \text {-a.e. } t \in J^{\kappa^{2}}, \\
-\left(u^{\Delta}\left(t_{j}^{+}\right)-u^{\Delta}\left(t_{j}^{-}\right)\right)=I_{j}\left(u\left(t_{j}\right)\right), \quad j=1,2, \ldots, p \\
u(0)=0=u(T)
\end{array}\right.
$$

where $f$ and $I_{j}, j=1, \ldots, p$ are continuous and $g$ is continuous and regressive.

We assume that $\lambda>-m \lambda_{1} / M$. Here, $m=\min _{t \in J} e_{g}(t, 0), M=\max _{t \in J} e_{g}(t, 0)$, where $e_{g}(t, 0)$ is an exponential function. Note that, as $g$ is regressive, $e_{g}(\cdot, 0)$ is the solution of the problem

$$
y^{\Delta}=g(t) y, \quad y(0)=1 .
$$

We transform the problem $(N P)$ into the following equivalent form:

$$
(N P E) \quad\left\{\begin{array}{l}
-\left(e_{g}(t, 0) u^{\Delta}(t)\right)^{\Delta}+\lambda e_{g}(t, 0) u^{\sigma}(t)=e_{g}(t, 0) f\left(t, u^{\sigma}(t)\right) ; \quad \Delta \text {-a.e. } t \in J^{\kappa^{2}}, \\
-\left(u^{\Delta}\left(t_{j}^{+}\right)-u^{\Delta}\left(t_{j}^{-}\right)\right)=I_{j}\left(u\left(t_{j}\right)\right), \quad j=1,2, \ldots, p, \\
u(0)=u(T)=0 .
\end{array}\right.
$$


Obviously, the solutions of (NPE) are solutions of $(N P)$. Consider the Hilbert space $H_{0, \Delta}^{1}(J)$ with the inner product:

$$
(u, v)=\int_{0}^{T} e_{g}(t, 0) u^{\Delta}(t) v^{\Delta}(t) \Delta t
$$

and the norm induced

$$
\|u\|=\left(\int_{0}^{T} e_{g}(t, 0)\left|u^{\Delta}(t)\right|^{2} \Delta t\right)^{\frac{1}{2}}
$$

A weak solution of $(N P E)$ is a function $u \in H_{0, \Delta}^{1}(J)$ such that

$$
\begin{aligned}
& \int_{0}^{T} e_{g}(t, 0) u^{\Delta}(t) v^{\Delta}(t) \Delta t+\lambda \int_{0}^{T} e_{g}(t, 0) u^{\sigma}(t) v^{\sigma}(t) \Delta t \\
& =\sum_{j=1}^{p} e_{g}\left(t_{j}, 0\right) I_{j}\left(u\left(t_{j}\right)\right) v\left(t_{j}\right)+\int_{0}^{T} e_{g}(t, 0) f\left(t, u^{\sigma}(t)\right) v^{\sigma}(t) \Delta t .
\end{aligned}
$$

Hence, a weak solution of $(N P)$ is a critical point of the following functional:

$$
\psi(u)=\frac{1}{2} A(u, u)-\sum_{j=1}^{p} e_{g}\left(t_{j}, 0\right) \int_{0}^{u\left(t_{j}\right)} I_{j}(t) d t-\int_{0}^{T} e_{g}(t, 0) F\left(t, u^{\sigma}(t)\right) \Delta t,
$$

where

$$
F(t, u)=\int_{0}^{u} f(t, \xi) d \xi
$$

and

$$
A(u, u)=\int_{0}^{T} e_{g}(t, 0) u^{\Delta}(t) v^{\Delta}(t) \Delta t+\lambda \int_{0}^{T} e_{g}(t, 0) u^{\sigma}(t) v^{\sigma}(t) \Delta t .
$$

It is evident that $A$ is bilinear, continuous and symmetric.

Lemma 4.1 (Theorem 38.A of [29]) For the functional $F: M \subset X \rightarrow \mathbb{R}$ with $M$ not empty, $\min _{u \in M} F(u)=a$ has solutions in case the following hold:

(i) $X$ is a reflexive Banach space.

(ii) $M$ is bounded and weak sequentially closed.

(iii) $\varphi$ is sequentially lower semi-continuous on $M$.

Lemma 4.2 (Analogous to Lemma 2.2 of [5]) There exist constants $\beta>\alpha>0$ such that

$$
\alpha\|u\|^{2} \leq A(u, u) \leq \beta\|u\|^{2}, \quad u \in H_{0, \Delta}^{1}(J) .
$$

Proof In fact, by Poincare's inequality, if $\lambda \geq 0$, we can take $\alpha=1, \beta=1+\frac{\lambda M}{\lambda_{1} m}$; if $\frac{-m \lambda_{1}}{M}<\lambda<$ 0 , then we can take $\alpha=1+\frac{\lambda M}{\lambda_{1} m}$ and $\beta=1$. 
Lemma 4.3 If $u \in H_{0, \Delta}^{1}(J)$, then there exists a constant $\delta>0$ such that $\|u\|_{0} \leq \delta\|u\|$, where

$$
\|u\|_{0}=\max _{t \in[0, T]_{\mathbb{T}}}|u(t)|
$$

Proof The result is followed by the following inequalities:

$$
\begin{aligned}
|u(t)| & \leq \int_{0}^{T}\left|u^{\Delta}(s)\right| \Delta s \leq\left(\int_{0}^{T} \frac{1}{e_{g}(t, 0)}\right)^{\frac{1}{2}}\left(\int_{0}^{T} e_{g}(t, 0)\left|u^{\Delta}(s)\right|^{2} \Delta s\right)^{\frac{1}{2}} \\
& \leq \sqrt{\frac{T}{m}}\|u\|=\delta\|u\| .
\end{aligned}
$$

Lemma 4.4 The functional $\psi$ defined by (4.1) is continuous, continuously differentiable and weakly lower semi-continuous.

Theorem 4.1 Suppose that $\lambda>\frac{-m \lambda_{1}}{M}, f$ and $I_{j}$ are bounded, $j=1,2, \ldots, p$, then $(N P)$ has at least one solution.

Proof Take $B>0$ and $B_{j}>0, j=1, \ldots, p$, such that

$$
\begin{aligned}
& |f(t, u)| \leq B \quad \forall(t, u) \in[0, T]_{\mathbb{T}} \times \mathbb{R} \\
& \left|I_{j}(u)\right| \leq B_{j} \quad \forall u \in \mathbb{R}, j=1,2, \ldots, p .
\end{aligned}
$$

For any $u \in H_{0, \Delta}^{1}(J)$, using Lemma 4.3 and Proposition 2.3, we have

$$
\psi(u) \geq \frac{\alpha}{2}\|u\|^{2}-\left(M \delta \sum_{j=1}^{p} B_{j}+B M \sqrt{\frac{T}{m \lambda_{1}}}\right)\|u\| .
$$

This implies that $\lim _{\|u\| \rightarrow \infty} \psi(u)=+\infty$, and $\psi$ is coercive. Hence, $\psi$ has a minimum, which is a critical point of $\psi$.

We will apply the mountain pass theorem in order to obtain at least two critical points of $\psi$.

Suppose that $X$ is a Banach space (in particular, a Hilbert space) and $\phi: X \rightarrow \mathbb{R}$ is differentiable and $c \in \mathbb{R}$. We say that $\phi$ satisfies the Palais-Smale condition if every bounded sequence $\left\{u_{k}\right\}$ in the space $X$ such that $\lim _{k \rightarrow \infty} \phi^{\prime}\left(u_{k}\right)=0$ contains a convergent subsequence.

Theorem 4.2 (Mountain pass theorem) Let $\phi \in \mathcal{C}^{1}$ be such that it satisfies the PalaisSmale condition. Assume that there exist $u_{0}, u_{1} \in X$ and a bounded neighborhood $\Omega$ of $u_{0}$ such that $u_{1} \notin \Omega$ and

$$
\inf \{\phi(u): u \in \partial \Omega\}>\max \left\{\phi\left(u_{0}\right), \phi\left(u_{1}\right)\right\} .
$$

Then there exists a critical point $u^{*}$ of $\phi$.

Theorem 4.3 Suppose that $\lambda>\frac{-m \lambda_{1}}{M}$, then the problem (NP) has at least two solutions if the following conditions hold: 
$\left(\mathrm{H}_{1}\right)$ There exist constants $\eta>2$ and $\gamma>0$ such that for all $(t, u) \in[0, T]_{\mathbb{T}} \times \mathbb{R},|u| \geq \gamma$

$$
0<\eta F(t, u) \leq u f(t, u), \quad 0<\eta \int_{0}^{u} I_{j}(\xi) d \xi \leq u I_{j}(u)
$$

where $j=1,2, \ldots, p$.

$\left(\mathrm{H}_{2}\right)$ There exists a positive $s \geq \eta$ such that $f(t, u)=o\left(|u|^{s}\right)$ and $I_{j}(u)=o\left(|u|^{s}\right)$ uniformly for $t \in[0, T]_{\mathbb{T}}$ as $|u| \rightarrow \infty, j=1,2, \ldots, p$.

$\left(\mathrm{H}_{3}\right) f(t, u)=o(|u|)$ and $I_{j}(u)=o(|u|)$ uniformly for $t \in[0, T]_{\mathbb{T}}$ as $|u| \rightarrow 0, j=1,2, \ldots, p$.

Proof From $\left(\mathrm{H}_{2}\right),\left(\mathrm{H}_{3}\right)$ and the continuities of $f$ and $I_{j}$, it is easy to see that for any $\varepsilon>0$ and $(t, u) \in[0, T]_{\mathbb{T}} \times \mathbb{R}$, there exist $C_{1}(\varepsilon)>0$ and $C_{1 j}>0$ such that

$$
\begin{aligned}
& |f(t, u)| \leq \varepsilon|u|+C_{1}(\varepsilon)|u|^{s}, \\
& \left|I_{j}(u)\right| \leq \varepsilon|u|+C_{1 j}(\varepsilon)|u|^{s} .
\end{aligned}
$$

Hence, for any $\varepsilon>0$ and $(t, u) \in[0, T]_{\mathbb{T}} \times \mathbb{R}$, we have

$$
\begin{aligned}
& F(t, u) \leq \int_{0}^{|u|}\left[\varepsilon \xi+C_{1}(\varepsilon) \xi^{s}\right] d \xi \leq \frac{\varepsilon}{2}|u|^{2}+C_{2}(\varepsilon)|u|^{s+1}, \\
& \int_{0}^{u} I_{j}(\xi) d \xi \leq \int_{0}^{|u|}\left[\varepsilon \xi+C_{1 j}(\varepsilon) \xi^{s}\right] d \xi \leq \frac{\varepsilon}{2}|u|^{2}+C_{2 j}(\varepsilon)|u|^{s+1}
\end{aligned}
$$

where $C_{2}(\varepsilon)=\frac{C_{1}(\varepsilon)}{s+1}$ and $C_{2 j}(\varepsilon)=\frac{C_{1 j}(\varepsilon)}{s+1}$.

From the condition $\left(\mathrm{H}_{1}\right)$, the following hold:

$$
\frac{\eta}{u} \leq \frac{f(t, u)}{F(t, u)} \quad \forall u \geq \gamma, \quad \frac{\eta}{u} \geq \frac{f(t, u)}{F(t, u)} \quad \forall u \leq-\gamma
$$

Integrating the above two inequalities with respect to $u$ on $[\gamma, u]$ and $[u,-\gamma]$, respectively (in this case, these are integrals on $\mathbb{R}$ ), we have

$$
\eta \ln \frac{u}{\gamma} \leq \ln \frac{F(t, u)}{F(t, \gamma)} \quad \forall u \geq \gamma, \quad \eta \ln \frac{\gamma}{-u} \geq \ln \frac{F(t,-\gamma)}{F(t, u)} \quad \forall u \leq-\gamma .
$$

That is,

$$
F(t, u) \geq F(t, \gamma)\left(\frac{u}{\gamma}\right)^{\eta} \quad \forall u \geq \gamma, \quad F(t, u) \geq F(t,-\gamma)\left(\frac{-u}{\gamma}\right)^{\eta} \quad \forall u \geq-\gamma .
$$

Thus there exists a constant $a_{1}>0$ such that $F(t, u) \geq a_{1}|u|^{\eta}$ for all $|u| \geq \gamma$.

From the continuity of $F(t, u)$, there exists a constant $k>0$ such that

$$
F(t, u) \geq-k \geq a_{1}|u|^{\eta}-a_{1} \gamma^{\eta}-k \quad \forall|u| \leq \gamma .
$$

Hence, we have

$$
F(t, u) \geq a_{1}|u|^{\eta}-a_{2} \quad \forall(t, u) \in[0, T]_{\mathbb{T}} \times \mathbb{R},
$$

where $a_{2}=a_{1} \gamma^{\eta}+k$. 
Similarly, there exist $a_{1 j}, a_{2 j}>0$ such that

$$
\int_{0}^{u} I_{j}(\xi) d \xi \geq a_{1 j}|u|^{\eta}-a_{2 j} \quad \forall u \in \mathbb{R}
$$

Firstly, we apply Lemma 4.1 to show that there exists $\rho$ such that $\psi$ has a local minimum $u_{0} \in B_{\rho}=\left\{u \in H_{0, \Delta}^{1}(J):\|u\|<\rho\right\}$.

Since $H_{0, \Delta}^{1}(J)$ is a Hilbert space, it is easy to deduce that $\bar{B}_{\rho}$ is bounded and weak sequentially closed. Lemma 4.4 has shown that $\psi$ is weak lower semi-continuous on $\bar{B}_{\rho}$ and, besides, $H_{0, \Delta}^{1}(J)$ is a reflexive Banach space. So, by Lemma 4.1 we can have this $u_{0}$ such that $\psi\left(u_{0}\right)=\min \left\{\psi(u): u \in \bar{B}_{\rho}\right\}$.

Now we will show that $\psi\left(u_{0}\right)=\min \left\{\psi(u): u \in \partial B_{\rho}\right\}$ for some $\rho=\rho_{0}$.

In fact, from (4.2) and (4.3), we obtain

$$
\begin{aligned}
\psi(u) \geq & \frac{\alpha}{2}\|u\|^{2}-\sum_{j=1}^{p} e_{g}(t, 0) \int_{0}^{u\left(t_{j}\right)} I_{j}(t) d t-\int_{0}^{T} e_{g}(t, 0) F\left(t, u^{\sigma}(t)\right) \Delta t \\
\geq & \frac{\alpha}{2}\|u\|^{2}-\sum_{j=1}^{p} e_{g}(t, 0)\left(\frac{\varepsilon}{2}|u|^{2}+C_{2 j}(\varepsilon)|u|^{s+1}\right) \\
& -\int_{0}^{T} e_{g}(t, 0)\left(\frac{\varepsilon}{2}|u|^{2}+C_{2}(\varepsilon)\left|u^{\sigma}\right|^{s+1}\right) \\
\geq & \frac{\alpha}{2}\|u\|^{2}-M \frac{\varepsilon}{2} p \delta^{2}\|u\|^{2}-\delta^{s+1} M \sum_{j=1}^{p} C_{2 j}(\varepsilon)\|u\|^{s+1} \\
& -\frac{M \varepsilon}{2} \int_{0}^{T}\left|u^{\sigma}(t)\right|^{2} \Delta t-M C_{2}(\varepsilon) \int_{0}^{T}\left|u^{\sigma}(t)\right|^{s+1} \Delta t \\
\geq & \frac{\alpha}{2}\|u\|^{2}-M \frac{\varepsilon}{2} p \delta^{2}\|u\|^{2}-\delta^{s+1} M \sum_{j=1}^{p} C_{2 j}(\varepsilon)\|u\|^{s+1} \\
& -\frac{M \varepsilon}{2} \frac{1}{m \lambda_{1}}\|u\|^{2}-M C_{2}(\varepsilon) \frac{T k^{s+1} c^{s+1}}{(\sqrt{m})^{s+1}}\|u\|^{s+1} .
\end{aligned}
$$

Hence,

$$
\psi(u) \geq \frac{\alpha-M \varepsilon\left(\frac{1}{m \lambda_{1}}+\delta^{2} p\right)}{2}\|u\|^{2}-M\left(\delta^{s+1} \sum_{j=1}^{p} C_{2 j}(\varepsilon)+\frac{C_{2}(\varepsilon) T k^{s+1} c^{s+1}}{(\sqrt{m})^{s+1}}\right)\|u\|^{s+1} .
$$

We can choose

$$
\begin{aligned}
& \varepsilon=\frac{\alpha}{2 M\left(\frac{1}{m \lambda_{1}}+\delta^{2} p\right)}, \\
& \rho_{0}=\left(\frac{\alpha}{8 M\left(\frac{T k^{s+1} c^{s+1}}{(\sqrt{m})^{s+1}} C_{2}(\varepsilon)+\delta^{s+1} \sum_{j=1}^{p} C_{2 j}(\varepsilon)\right)}\right)^{\frac{1}{s-1}} .
\end{aligned}
$$

For any $u \in \partial B_{\rho_{0}},\|u\|=\rho_{0}$, we have $\psi(u) \geq \frac{\alpha}{8 \rho_{0}^{2}}>0$. Besides, $\psi\left(u_{0}\right) \leq \psi(0)=0$. Then $\psi(u)>\frac{\alpha}{8 \rho_{0}^{2}}>\psi(0) \geq \psi\left(u_{0}\right)$ for any $u \in \partial B_{\rho_{0}}$. So, $\psi\left(u_{0}\right)<\inf \left\{\psi(u): u \in \partial B_{\rho_{0}}\right\}$. Hence, $\psi$ has a local minimum $u_{0} \in B_{\rho_{0}}=\left\{u \in H_{0, \Delta}^{1}(J):\|u\|<\rho_{0}\right\}$. 
Next, we will show that there exists $u_{1}$ with $\left\|u_{1}\right\|>\rho_{0}$ such that $\psi\left(u_{1}\right)<\inf \{\psi(u): u \in$ $\left.\partial B_{\rho_{0}}\right\}$.

From (4.4) and (4.5), we have

$$
\begin{aligned}
\psi(u) & \leq \frac{\beta}{2}\|u\|^{2}-\sum_{j=1}^{p} e_{g}(t, 0) \int_{0}^{u\left(t_{j}\right)} I_{j}(t) d t-\int_{0}^{T} e_{g}(t, 0) F\left(t, u^{\sigma}(t)\right) \Delta t \\
& \leq \frac{\beta}{2}\|u\|^{2}-\sum_{j=1}^{p} e_{g}(t, 0)\left(a_{1 j}\left|u\left(t_{j}\right)\right|^{\eta}-a_{2 j}\right)-\int_{0}^{T} e_{g}(t, 0)\left(a_{1}\left|u^{\sigma}(t)\right|^{\eta}-a_{2}\right) \\
& \leq \frac{\beta}{2}\|u\|^{2}-m \sum_{j=1}^{p} a_{1 j}\left|u\left(t_{j}\right)\right|^{\eta}+M \sum_{j=1}^{p} a_{2 j}-m a_{1} \int_{0}^{T}\left|u^{\sigma}(t)\right|^{\eta} \Delta t+M a_{2} T .
\end{aligned}
$$

Thus,

$$
\psi(u) \leq \frac{\beta}{2}\|u\|^{2}-m \sum_{j=1}^{p} a_{1 j}\left|u\left(t_{j}\right)\right|^{\eta}+M \sum_{j=1}^{p} a_{2 j}-m a_{1}\left\|u^{\sigma}\right\|_{L_{\Delta}^{\eta}}^{\eta}+M a_{2} T .
$$

For any $u \in H_{0, \Delta}^{1}(J)$ with $\|u\|=1$, we have

$$
\psi(N u) \leq \frac{\beta}{2} N^{2}-m \sum_{j=1}^{p} a_{1 j} N^{\eta}\left|u\left(t_{j}\right)\right|^{\eta}+M \sum_{j=1}^{p} a_{2 j}-m a_{1} N^{\eta}\left\|u^{\sigma}\right\|_{L_{\Delta}^{\eta}}+M a_{2} T .
$$

So, $\lim _{N \rightarrow \infty} \psi(N u)=-\infty$ since $\eta>2$. Then there exists $N_{0}>\rho_{0}$ such that $\psi\left(N_{0} u\right) \leq 0$.

Hence, for the above $\rho_{0}$, there exists $u_{1}$ such that $\left\|u_{1}\right\|=N_{0}$ and $\psi\left(u_{1}\right)<0$.

Then, we have $\max \left\{\psi\left(u_{0}\right), \psi\left(u_{1}\right)\right\}<\inf \left\{\psi(u): u \in \partial B_{T_{0}}\right\}$.

The next step is to show that $\psi$ satisfies the Palais-Smale condition.

Let $\left\{\psi\left(u_{k}\right)\right\}$ be a bounded sequence such that $\lim _{k \rightarrow \infty} \psi^{\prime}\left(u_{k}\right)=0$. Now we show that $\left\|u_{k}\right\|$ is bounded. By (4.1) we have

$$
\begin{aligned}
\left(\psi^{\prime}\left(u_{k}\right), u_{k}\right)= & A\left(u_{k}, u_{k}\right)-\int_{0}^{T} e_{g}(t, 0) f\left(t, u_{k}^{\sigma}(t)\right) u_{k}^{\sigma}(t) \Delta t \\
& -\sum_{j=1}^{p} e_{g}(t, 0) I_{j}\left(u_{k}\left(t_{j}\right)\right) u_{k}\left(t_{j}\right) .
\end{aligned}
$$

Thus,

$$
\begin{aligned}
\psi\left(u_{k}\right)-\frac{1}{\eta}\left(\psi^{\prime}\left(u_{k}\right), u_{k}\right) & =\left(\frac{1}{2}-\frac{1}{\eta}\right) A\left(u_{k}, u_{k}\right)+\Gamma_{1}+\Gamma_{2} \\
& \geq\left(\frac{1}{2}-\frac{1}{\eta}\right) \alpha\left\|u_{k}\right\|^{2}+\Gamma_{1}+\Gamma_{2},
\end{aligned}
$$

where

$$
\begin{aligned}
& \Gamma_{1}=\frac{1}{\eta} \int_{0}^{T} e_{g}(t, 0) f\left(t, u_{k}^{\sigma}(t)\right) u_{k}^{\sigma}(t) \Delta t-\int_{0}^{T} e_{g}(t, 0) F\left(t, u_{k}^{\sigma}(t)\right) \Delta t, \\
& \Gamma_{2}=\frac{1}{\eta} \sum_{j=1}^{p} e_{g}(t, 0) I_{j}\left(u_{k}\left(t_{j}\right)\right) u_{k}\left(t_{j}\right)-\sum_{j=1}^{p} e_{g}(t, 0) \int_{0}^{u_{k}\left(t_{j}\right)} I_{j}(t) d t .
\end{aligned}
$$


Note that $J=\Upsilon_{1}^{k} \cup \Upsilon_{2}^{k}$, where $\Upsilon_{1}^{k}=\left\{t \in J:\left|u_{k}(t)\right|<\gamma\right\}, \Upsilon_{2}^{k}=\left\{t \in J:\left|u_{k}(t)\right| \geq \gamma\right\}$, and that there exists a constant $c$ such that

$$
\begin{aligned}
& \left|F\left(t, u^{\sigma}(t)\right)\right| \leq c, \quad\left|f\left(t, u^{\sigma}(t) u^{\sigma}(t)\right)\right| \leq c, \quad \text { if }|u|<\gamma, \\
& \left|\int_{0}^{u\left(t_{j}\right)} I_{j}(t) d t\right| \leq c, \quad\left|I_{j}\left(u\left(t_{j}\right)\right) u\left(t_{j}\right)\right| \leq c, \quad \text { if }|u|<\gamma .
\end{aligned}
$$

So, by $\left(\mathrm{H}_{1}\right)$ and (4.7), we have

$$
\begin{aligned}
\Gamma_{1}= & \frac{1}{\eta} \int_{\Upsilon_{1}^{k}} e_{g}(t, 0) f\left(t, u_{k}^{\sigma}(t)\right) u_{k}^{\sigma}(t) \Delta t+\frac{1}{\eta} \int_{\Upsilon_{2}^{k}} e_{g}(t, 0) f\left(t, u_{k}^{\sigma}(t)\right) u_{k}^{\sigma}(t) \Delta t \\
& -\int_{\Upsilon_{1}^{k}} e_{g}(t, 0) F\left(t, u_{k}^{\sigma}(t)\right) \Delta t-\int_{\Upsilon_{2}^{k}} e_{g}(t, 0) F\left(t, u_{k}^{\sigma}(t)\right) \Delta t \\
\geq & -\frac{1}{\eta} \int_{\Upsilon_{1}^{k}} e_{g}(t, 0)\left|f\left(t, u_{k}^{\sigma}(t)\right) u_{k}^{\sigma}(t)\right| \Delta t-\int_{\Upsilon_{1}^{k}} e_{g}(t, 0)\left|F\left(t, u_{k}^{\sigma}(t)\right)\right| \Delta t \\
& +\frac{1}{\eta} \int_{\Upsilon_{2}^{k}} e_{g}(t, 0) f\left(t, u_{k}^{\sigma}(t)\right) u_{k}^{\sigma}(t) \Delta t-\int_{\Upsilon_{2}^{k}} e_{g}(t, 0) F\left(t, u_{k}^{\sigma}(t)\right) \Delta t \\
\geq & c^{\prime}+c^{\prime \prime}=c_{1},
\end{aligned}
$$

where $c^{\prime}, c^{\prime \prime}$ and $c_{1}$ are constants (independent of $k$ ).

Analogously, there exists a constant $c_{2}$ (independent of $k$ ) such that $\Upsilon_{2}^{k} \geq c_{2}$.

Hence,

$$
\begin{aligned}
\psi\left(u_{k}\right) & \geq\left(\frac{1}{2}-\frac{1}{\eta}\right) \alpha\left\|u_{k}\right\|^{2}+\frac{1}{\eta}\left(\psi^{\prime}\left(u_{k}\right), u_{k}\right)+\Gamma_{1}+\Gamma_{2} \\
& \geq\left(\frac{1}{2}-\frac{1}{\eta}\right) \alpha\left\|u_{k}\right\|^{2}-\frac{1}{\eta}\left\|\psi^{\prime}\left(u_{k}\right)\right\|\left\|u_{k}\right\|+c_{1}+c_{2} .
\end{aligned}
$$

Since $\psi\left(u_{k}\right)$ is bounded, we have $\left\{\left\|u_{k}\right\|\right\}_{k=1}^{\infty}$ is a bounded sequence.

Hence, there exists a subsequence $\left\{u_{k}\right\}$ (for simplicity denoted again by $\left\{u_{k}\right\}$ ) such that $\left\{u_{k}\right\}$ weakly converges to some $u$ in $H_{0, \Delta}^{1}(J)$. Then the sequence $\left\{u_{k}\right\}$ converges uniformly to $u$ in $\mathcal{C}(J)$.

By (4.6), we have

$$
\begin{aligned}
\left\|u_{k}\right\|^{2}= & \int_{0}^{T} e_{g}(t, 0)\left(f\left(t, u_{k}^{\sigma}\right) u_{k}^{\sigma}-\lambda u_{k}^{2}\right) \Delta t+\left(\psi^{\prime}\left(u_{k}\right), u_{k}\right) \\
& +\sum_{j=1}^{p} e_{g}(t, 0) I_{j}\left(u_{k}\left(t_{j}\right)\right) u_{k}\left(t_{j}\right) .
\end{aligned}
$$

So, we have

$$
\lim _{k \rightarrow \infty}\left\|u_{k}\right\|^{2}=\int_{0}^{T} e_{g}(t, 0)\left(f\left(t, u^{\sigma}\right) u^{\sigma}-\lambda u^{2}\right) \Delta t+\sum_{j=1}^{p} e_{g}(t, 0) I_{j}\left(u\left(t_{j}\right)\right) u\left(t_{j}\right) .
$$

Then $\left\|u_{k}\right\|$ converges in $H_{0, \Delta}^{1}(J)$. Since $H_{0, \Delta}^{1}(J)$ is a Hilbert space, and the sequence $\left\{u_{k}\right\} \in$ $H_{0, \Delta}^{1}(J)$ satisfies $u_{k} \rightarrow u$, then $\left\{u_{k}\right\}$ converges to $u$, i.e., $u_{k} \rightarrow u$. $\psi$ satisfies the Palais-Smale condition. 
Now, by Theorem 4.2 , there exists a critical point $u^{*}$. Therefore, $u_{0}$ and $u^{*}$ are two critical points of $\psi$, and they are classical solutions of $(N P E)$. Hence, $u_{0}$ and $u^{*}$ are classical solutions of $(N P)$.

Example 4.1 Let $\mathbb{T}=h \mathbb{Z}$ for $0<h<T, T \in h \mathbb{Z}$ and $t_{1} \in(0, T)$. Thus, $J=[0, T] \cap h \mathbb{Z}$ and $J^{\kappa}=[0, T-h] \cap h \mathbb{Z}$. Consider the following boundary value problem:

$$
\left\{\begin{array}{l}
-u^{\Delta \Delta}(t)+\alpha u^{\Delta}(t+h)+\lambda u(t+h)=t u^{5}(t+h) ; \quad \Delta \text {-a.e. } t \in J^{\kappa^{2}}, \\
-\left(u^{\Delta}\left(t_{1}^{+}\right)-u^{\Delta}\left(t_{1}^{-}\right)\right)=u^{5}\left(t_{1}\right), \\
u(0)=0=u(T),
\end{array}\right.
$$

where $\alpha>0$ is a constant.

We can see that $g(t)=\alpha$ is regressive and continuous. If we take $\eta=s=6$ and $\lambda>\frac{-\lambda_{1}}{(1+\alpha h)^{\frac{T}{h}}}$, by Theorem 4.3, Eq. (4.9) has at least two solutions.

\section{Competing interests}

The authors declare that they have no competing interests.

\section{Authors' contributions}

All authors contributed equally in this article. They read and approved the final manuscript.

\section{Author details}

'Departamento de Análise Matemática, Universidade de Santiago de Compostela, Santiago de Compostela, Galicia 15782, Spain. ${ }^{2}$ Instituto de Ciencias Matemáticas (CSIC, UAM, UC3M, UCM), Madrid, 28049, Spain.

\section{Acknowledgements}

The authors are grateful to the referees for their valuable suggestions that led to the improvement of the original manuscript. The research of $\vee$ Otero-Espinar has been partially supported by Ministerio de Educación y Ciencia (Spain) and FEDER, Project MTM2010-15314.

\section{Received: 27 February 2013 Accepted: 23 April 2013 Published: 9 May 2013}

\section{References}

1. Jankowski, T: Positive solutions to second order differential equations with dependence on the first order derivative and nonlocal boundary conditions. Bound. Value Probl. 2013, 8 (2013). doi:10.1186/1687-2770-2013-8

2. Kaufmann, ER, Kosmatov, N, Raffoul, YN: A second-order boundary value problem with impulsive effects on an unbounded domain. Nonlinear Anal. 69(9), 2924-2929 (2008)

3. Guo, Y, Ge, W: Positive solutions for three-point boundary value problems with dependence on the first order derivative. J. Math. Anal. Appl. 290(1), 291-301 (2004)

4. Nieto, JJ, O'Regan, D: Variational approach to impulsive differential equations. Nonlinear Anal., Real World Appl. 10(2), 680-690 (2009)

5. Xiao, J, Nieto, JJ: Variational approach to some damped Dirichlet nonlinear impulsive differential equations. J. Franklin Inst. 348(2), 369-377 (2011)

6. Xiao, J, Nieto, JJ, Luo, Z: Multiplicity of solutions for nonlinear second order impulsive differential equations with linear derivative dependence via variational methods. Commun. Nonlinear Sci. Numer. Simul. 17(1), 426-432 (2012)

7. Xian, X, O'Regan, D, Agarwal, RP: Multiplicity results via topological degree for impulsive boundary value problems under non-well-ordered upper and lower solution conditions. Bound. Value Probl. 2008, Article ID 197205 (2008)

8. Yan, B, O'Regan, D, Agarwal, RP: Multiple positive solutions of singular second order boundary value problems with derivative dependence. Aequ. Math. 74(1-2), 62-89 (2007)

9. Martins, N, Torres, DFM: Necessary optimality conditions for higher-order infinite horizon variational problems on time scales. J. Optim. Theory Appl. 155(2), 453-476 (2012)

10. Agarwal, RP, Franco, D, O’Regan, D: Singular boundary value problems for first and second order impulsive differential equations. Aequ. Math. 69(1-2), 83-96 (2005)

11. Sun, H-R, Li, Y-N, Nieto, JJ, Tang, Q: Existence of solutions for Sturm-Liouville boundary value problem of impulsive differential equations. Abstr. Appl. Anal. 2012, Article ID 707163 (2012)

12. Benchohra, M, Ntouyas, SK, Ouahab, A: Extremal solutions of second order impulsive dynamic equations on time scales. J. Math. Anal. Appl. 324(1), 425-434 (2006)

13. Chen, $\mathrm{H}$, Wang, $\mathrm{H}$ : Triple positive solutions of boundary value problems for $p$-Laplacian impulsive dynamic equations on time scales. Math. Comput. Model. 47(9-10), 917-924 (2008)

14. Kaufmann, ER: Impulsive periodic boundary value problems for dynamic equations on time scale. Adv. Differ. Equ. 2009, Article ID 603271 (2009) 
15. Agarwal, RP, Otero-Espinar, V, Perera, K, Vivero, DR: Basic properties of Sobolev's spaces on time scales. Adv. Differ. Equ. 2006, Article ID 38121 (2006)

16. Agarwal, RP, Otero-Espinar, V, Perera, K, Vivero, DR: Existence of multiple positive solutions for second order nonlinear dynamic BVPs by variational methods. J. Math. Anal. Appl. 331(2), 1263-1274 (2007)

17. Agarwal, RP, Otero-Espinar, V, Perera, K, Vivero, DR: Multiple positive solutions of singular Dirichlet problems on time scales via variational methods. Nonlinear Anal. 67(2), 368-381 (2007)

18. Zhou, J, Li, Y: Variational approach to a class of second order Hamiltonian systems on time scales. Acta Appl. Math. $117,47-69(2012)$

19. Zhou, J, Wang, Y, Li, Y: Existence and multiplicity of solutions for some second-order systems on time scales with impulsive effects. Bound. Value Probl. 2012, 148 (2012)

20. Duan, H, Fang, H: Existence of weak solutions for second-order boundary value problem of impulsive dynamic equations on time scales. Adv. Differ. Equ. 2009, Article ID 907368 (2009)

21. Bohner, M, Peterson, A: Dynamic Equations on Time Scales: An Introduction with Applications. Birkhäuser, Boston (2001)

22. Cieśliński, JL: New definitions of exponential, hyperbolic and trigonometric functions on time scales. J. Math. Anal. Appl. 388(1), 8-22 (2012)

23. Slavík, A: Averaging dynamic equations on time scales. J. Math. Anal. Appl. 388(2), 996-1012 (2012)

24. Slavík, A: Dynamic equations on time scales and generalized ordinary differential equations. J. Math. Anal. Appl. 385(1), 534-550 (2012)

25. Rabinowitz, PH: Minimax Methods in Critical Point Theory with Applications to Differential Equations. CBMS Regional Conference Series in Mathematics, vol. 65 (1986). Published for the Conference Board of the Mathematical Sciences, Washington, DC

26. Mawhin, J, Willem, M: Critical Point Theory and Hamiltonian Systems. Applied Mathematical Sciences, vol. 74. Springer, New York (1989)

27. Agarwal, RP, Otero-Espinar, V, Perera, K, Vivero, DR: Wirtinger's inequalities on time scales. Can. Math. Bull. 51(2), 161-171 (2008)

28. Cresson, J, Malinowska, AB, Torres, DFM: Time scale differential, integral, and variational embeddings of Lagrangian systems. Comput. Math. Appl. 64(7), 2294-2301 (2012)

29. Zeidler, E: Nonlinear Functional Analysis and Its Applications. III: Variational Methods and Optimization. Springer, New York (1985). Translated from the German by Leo F. Boron

doi:10.1186/1687-2770-2013-119

Cite this article as: Otero-Espinar and Pernas-Castaño: Variational approach to second-order impulsive dynamic equations on time scales. Boundary Value Problems 2013 2013:119.

\section{Submit your manuscript to a SpringerOpen ${ }^{\circ}$ journal and benefit from:}

- Convenient online submission

- Rigorous peer review

- Immediate publication on acceptance

- Open access: articles freely available online

- High visibility within the field

- Retaining the copyright to your article 